\title{
A Visibility Restoration Algorithm for Real-World Hazy Scenes
}

\author{
Sanjay Sharma \\ Shri Shankaracharya Technical \\ Campus, Bhilai
}

\author{
Padma J. Bonde, PhD \\ Shri Shankaracharya Technical \\ Campus, Bhilai
}

\author{
Samta Gajbhiye \\ Shri Shankaracharya Technical \\ Campus, Bhilai
}

\begin{abstract}
With the increase in industrial production and human activities, the concentration of atmospheric particulate matter (PM) is substantially increased; due to which fog and haze occur more frequently. Limited visibility is caused by suspended particles in the air, such as fog and haze are a major problem for many applications of computer vision. The captured scenes by such computer vision systems suffer from poor visibility, low contrast, dimmed brightness, low luminance and distorted color. The detection of objects within the scene is more difficult. Therefore visibility improvement, contrast and features enhancement of images and videos captured in bad weather are also called as dehazing, is an inevitable task.

The Motion detection is the first essential process within the extraction of data concerning moving objects and makes use of stabilization in purposeful areas, like tracking, classification, recognition, and so on. A total unique and accurate approach to motion detection for the automated video surveillance system has been adopted. Complete detection of moving objects can be achieved by involving three significant projected units, a background modeling (BM) unit, an alarm trigger (AT) unit and an object extraction (OE) module. Intelligent service mechanism development is a crucial and critical issue for human community applications. With the diverse and complicated service desires, the perception and navigation are essential subjects. First of all, a new augmented approach of graph-based optimum estimation derived for concurrent mechanism postures and affecting objective course approximation. Moreover, all the moving object detection issues of a robot's indoor navigation has been solved by divided and conquered via multisensory fusion methodologies.
\end{abstract}

\section{Keywords}

Retinex theory, Visibility restoration, dehazing, Edgepreserving regularization, Fusion technique.

\section{INTRODUCTION}

Outdoor pictures captured in weather condition (foggy or hazy), sometimes low contrast and fidelity, resulting poor visibility of the objects, because of light absorbed and scattered by the turbid medium like particles and water droplets within the atmosphere during propagation. Moreover, most automatic systems were strongly depended upon the definition of the participation images and unsuccessful to work typically reason by the tainted images. Therefore, the technique of haze removal can benefit in several image understanding and pc-vision applications like aerial imagery [1], image classification [2]-[5], image or video retrieval [6]-[8], remote sensing and video analysis and recognition [12]-[14].

Since concentration of the haze was completely different from place to place and it was tough to find during a hazy image , hence dehazing is a difficult task. Initially researchers were used the conventional techniques of image process to remove the haze from a single image (Histogram-based dehazing strategies). However, the dehazing result was restricted. As a result of a single hazy image will hardly give a lot of information. Later, researchers tried and improved the dehazing performance with multiple pictures.

Polarization based ways in [15] were used for dehazing with multiple pictures which were taken with completely different degrees of polarization and proposed haze removal approaches under different atmospheric condition. Dehazing was based on the given depth information. Recently, significant progress has been achieved in single image dehazing which based on the physical model. Under the assumption, that the local contrast of the haze-free image is much higher than that in the hazy image. [16] has been used a haze removal method by maximizing the local contrast of the image, supported in Markov Random Field (MRF).Although, it has observed the spectacular results given over-saturated pictures. [17] has been proposed independent component analysis (ICA) to remove the haze from color pictures.

However, the approach was time-consuming and can't be used for grayscale image dehazing. Moreover, it was quite difficult to deal with dense-haze images, inspired by the widely used dark-object subtraction technique based on a large variety of experiments on haze-free images, discovered the dark channel prior (DCP) that, in most of the non-sky patches, at least one color channel has some pixels whose intensities were very low and close to zero. With this prior, they estimate the thickness of haze, and restore the haze-free image in the atmospherically scattering model.

\section{LITERATURE SURVEY}

Images captured in [1] were affected with degraded visibility and undesirable color forged effects in sandstorm situation. In such circumstances, conventional visibility restoration approaches could not be adequately restore pictures due to poor estimation of haze thickness and the persistence of color cast issues. A complete unique Laplacian-based visibility restoration approach was used to effectively solve inadequate haze width judgment, color cast problems and restoration of degraded picture captured throughout the atmospheric condition, like fog and sandstorms, was used. The fig.1 depicted in proposed HTE module.

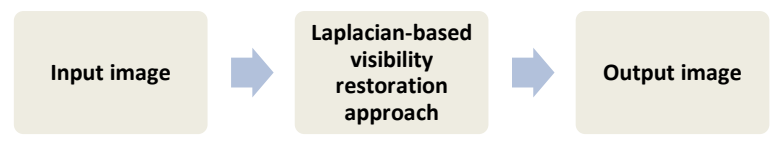

Fig.1 Laplacian-based Visibility Restoration Method

The proposed technique discussed [1] is Laplacian distribution model and a mixture of the projected HTE module along with the proposed IVR module used to remove haze formation and consequently recovered a vivid colorized 
picture. Laplacian-based gamma correction model, employed during the projected HTE module, used to refine an insufficient transmission map and therefore, accomplished effective estimation of haze thickness, after which the refined transmission map was used to restore the visibility of a degraded image via the projected IVR module. The projected IVR module utilized Laplacian based mostly white patchRetinex theory was used to equalize every RGB color channel and subsequently over come color cast problems in restored pictures. Consequently, the proposed IVR module effectively generated haze-free and vivid restoration results for degraded pictures captured during varied atmospheric condition.

\section{Limitation:}

These existing methods with multiple reference images have limitations in online dehazing applications and may need a special imaging sensor [12]. It provided a lead the researchers to focus the dehazing method with a single reference image. Single image based methods would rely on the typical characteristics of haze-free images.

A single image dehazing in [2] was challenging because it was massively ill-posed. There was used dark channel prior to remove haze, but refining the transmission map with closedform matting was computationally expensive. Recent work could be refined the transmission map with guided filter to achieve feasibility. A single image dehazing has been solved by combining dark channel prior technique and guided image filtering method. By analyzing the tradeoffs of this approach, it proposed an effective scheme to adapt the parameters like $p$ as an input image, I as a guidance image and $\mathrm{q}$ as a filtering output.

A single dehazing image was elaborated by combining dark channel prior and guided image filtering and studied several aspects of this approach. The method has generated satisfactory dehazed results with low computation by comparative analysis and experiments. It proposed an effective method to adapt the patch size of dark channel and the filtering radius of guided filter. The main benefit of using guided filter was to refine the transmission map and low computational cost. It has generated comparatively dehazed results. Since a guided image filtering was an approximation of soft matting. This method may fail when the input image contains abrupt depth changes. Fortunately, it turned out, work performance was quite well on many hazy images, its $\mathrm{O}(\mathrm{N})$ time complexity was also appealed in many applications. The advantage of combining dark channel prior and guided image filter was low computational cost to dehaze the images.

Many image processes in [3] faced problems and must be regularized. Usually, roughness penalty imposed on the solution. The difficulty is to avoid the smoothing of edges. That is an important attribute of the image. It has provided conditions for the design of an edge-preserving regularization. Under these conditions, it was possible to introduce an auxiliary variable whose role was twofold. First, it marked the discontinuities and ensured their perpetuation from flat $\&$ the criterion half-quadratic.

The optimization became easier and discussed a deterministic strategy based on alternate minimizations on the image and the auxiliary variable. It consequently results in the definition of an ingenious reconstruction algorithmic rule ARTUR. A number of theoretical properties of ARTUR were discussed. Experimental results were illustrated the behavior of the algorithmic rule. These results are shown in the field of tomography, still this technique applied in a large number of applications in image processing. Here, it is observed that edge-preserving regularization is a problem in computed image. There were three conditions for edge preservation further limitation in [3] is large gradients due to noise.

Image fusion method discussed in [4], component of data fusion, when data type was resist to image format. Image fusion was an effective way for optimum utilization of large volumes of image from multiple sources

A good fused image has a good quality. So the grouping of DWT and spatial domain combination method (PCA) enhanced performance as compare to use of individual DWT and PCA algorithm. But, this method is complex in fusion algorithm. A good fusion technique is used for better result. The fusion algorithm may be optimized for speed up in future research.

Paper [5] has centered on the degraded document binarization technique. Document binarization was a chief application of vision process. The main aim of this paper was to evaluate the short comings of algorithms for degraded image binarization. It is found that every technique has its own advantages and limitations. No technique was the best for each case. The main limitations of [5] were found that the images were noisy and low intensity. To beat the short comings of previous work, a new algorithm has been designed which used additional reliable methodology to get better result of the work. The proposed algorithm used the guided image filter to enhance the results for noisy images. The newly developed algorithm outperformed over the available methods and comparative analysis result has been shown there. The main limitations of this work are that the resultant images were noisy and low intensity.

\section{METHOD}

\subsection{Retinex Theory}

Retinex is independent of the flux of radiant energy due to the reflection factor of objects and must be treated as a color code for a three-part report from the retina. There are still left some work without a satisfactory explanation of it. The modern technology of color photography has reinforced the belief that the colors within the spectrum were discerned.

A daylight colored film taken under tungsten-filament lamp became reddish, since the rays from the tungsten filament were too red. In determining color, extra red was never perceived because it is independ on the flux of radiant energy reaching to it.

\subsection{Visibility Restoration}

Visibility restoration has been designed to decrease or remove the degradation that occurred in digital image. The degradation may be due to various factors like camera motions relative to object, blur due to camera misfocus, relative atmospherically turbulence etc. It was discussed in [1] about the degradations due to weather conditions like fog, haze, rain and snow in a picture.

The image quality of outside screen in the fog and haze, atmospheric phenomenon, was sometimes degraded by the scattering of a light before reaching the camera or due to these large quantities of suspended particles (e.g. fog, haze, smoke, impurities) in the atmosphere. This phenomenon has been affected the normal work of automatic watching system, outside recognition system and intelligent transportation. Scattering was caused by two elementary phenomena like attenuation and air light. It is simply to improve the steadiness and hardiness of the visual system. There were many methods 
to remove haze, fog, mist, from the given image. Some strategies of image restoration of the fog are:

- Dark channel prior

- Clahe

- Wiener filtering

- Bilateral filtering

The limitations of the above reviewed papers are:

Image quality under various weather conditions was poor, high complexity and processing time.

\subsection{Edge-Preserving Regularization}

It was difficult to remove rough-area from the edges of input images. Under these conditions, an auxiliary variable has been used, which is very important attribute of the image and having the two roles. First, it marked the discontinuities and ensured their preservation from smoothing. Secondly, it marked criterion half-quadratic. Therefore, the optimization was then easier and proposed a deterministic strategy based on alternate minimizations of the image and the auxiliary variable. It is helpful to create an original algorithm ARTUR.

\subsection{Fusion Technique}

Image fusion technique combined the relevant information from a set of images into a single image. The resultant fused image became more informative and complete than any of the input images. Image fusion techniques could be improved the quality and increased the area of applications. Some of the important applications of the fusion image are defined in medical imaging, microscopic imaging, remote sensing, computer vision, and robotics.Image fusion method can be broadly classified into two groups

$$
\begin{aligned}
& \text { - } \quad \text { Spatial domain fusion method } \\
& \text { Transform domain fusion }
\end{aligned}
$$

\section{CONCLUSION}

Several haze removal techniques have been reviewed. It is observed that Retnix theory enhances the color quality and visibility restoration to remove degradation from input image. Edge preserving regularization is used to remove roughness from edges of the input image while image fusion technique is improved the quality of the image. It is concluded that above methods performed well in certain but not covered all aspects which enhance image quality, hence it is needed one solution which will covered all issues which degrades the quality.

\section{REFERENCES}

[1] Shih-Chia Huang, Jian-Hui Ye, and Bo-Hao Chen "An Advanced Single-Image Visibility Restoration Algorithm for Real-World Hazy Scenes" IEEE Trans.on industrial electronics, vol. 62, no. 5, may 2015

[2] Jiahao Pang, Oscar C. Au and Zheng Guo "Improved Single Image Dehazing Using Guided Filter", APSIPA ASC 2011 Xi'an

[3] Pierre Charbonnier, Laure Blanc-Feraud, Gilles Aubert, and Michel Barlaud, "Deterministic Edge-Preserving Regularization in Computed Imaging", IEEE Trans. On image processing, vol. 6, no. 2, FEBRUARY 1997.

[4] Anju Rani, Gagandeep Kaur "Image Enhancement using Image Fusion Techniques", IJARCSSE volume 4,Issue 9 September - 2014, pp. 413-416.
[5] Er. Jagroop Kaur, Dr. Rajiv Mahajan "Improved Degraded Document Image Binarization Using Guided Image Filter", IJARCSSE, Volume 4, Issue 9, September 2014

[6] R. C. Luo and C. L. Chun, "Multisensor fusion-based concurrent environment mapping and moving object detection for intelligent service robotics," IEEE Trans. Ind. Electron., vol. 61, no. 8, pp. 4043-4051,Aug. 2014.

[7] H. Zhuang, K. S. Low, and W.Y.Yau, "Multichannel pulse-coupledneuralnetwork-based color image segmentation for object detection," IEEETrans. Ind. Electron., vol. 59, no. 8, pp. 3299-3308, Aug. 2012.

[8] H. H. Kim, D. J. Kim, and K. H. Park, "Robust elevator button recognitionin the presence of partial occlusion and clutter by specular reflections,"IEEE Trans. Ind. Electron., vol. 59, no. 3, pp. 1597-1611, Mar. 2012.

[9] H. Rezaee and F. Abdollahi, "A decentralized cooperative control scheme with obstacle avoidance for a team of mobile robots," IEEE Trans. Ind.Electron., vol. 61, no. 1, pp. 347-354, Jan. 2014

[10] J. S. Hu, J. J. Wang, and D. M. Ho, "Design of sensing system and anticipative behavior for human following of mobile robots," IEEE Trans. Ind. Electron., vol. 61, no. 4, pp. 1916-1927, Apr. 2014.

[11] S. Hong, Y. Oh, D. Kim, and B. J. You, "Real-time walking pattern generation method for humanoid robots by combining feedback and feedforward controller," IEEE Trans. Ind. Electron., vol. 61, no. 1, pp. 355-364, Jan. 2014

[12] Lee, Sungmin, et al. "A review on dark channel prior based image dehazing algorithms." EURASIP Journal on Image and Video Processing 2016.1 (2016): 4.

[13] S. C. Huang, "An advanced motion detection algorithm with video quality analysis for video surveillance systems," IEEE Trans. Circuits Syst. Video Technol., vol. 21, no. 1, pp. 1-14, Jan. 2011.

[14] X. Zhang, W. Hu, S. Chen, and S. Maybank, "Graphembedding-based learning for robust object tracking," IEEE Trans. Ind. Electron., vol. 61, no. 2, pp. 1072 1084, Feb. 2014

[15] Y. Y. Schechner, S. G. Narasimhan, and S. K. Nayar, "Polarization based vision through haze," Appl. Opt., vol. 42, no. 3, pp. 511-525, Jan. 2003.

[16] K. Tan and J. P. Oakley, "Enhancement of color images in poor visibility conditions," in Proc. IEEE ICIP, Sep. 2000, vol. 2, pp. 788-791.

[17] R. Fattal, "Single image dehazing," in Proc. ACM SIGGRAPH, 2008, pp. 1-7.

[18] S. C. Huang and B. H. Chen, "Highly accurate moving object detection in variable-bit-rate video-based traffic monitoring systems," IEEE Trans. Neural Netw. Learn. Syst., vol. 24, no. 12, pp. 1920-1931, Dec. 2013.

[19] M. Chacon and S. Gonzalez, "An adaptive neural-fuzzy approach for object detection in dynamic backgrounds for surveillance systems," IEEE Trans. Ind. Electron., vol. 59, no. 8, pp. 3286-3298, Aug. 2012. 